\title{
A Generalization and Simple Proof of Kronecker's Theorem* Concerning the Minors of a Compound Determinant.
}

\author{
By Professor W. H. Metzlar.
}

(Received 18th April 1924. Read 2nd May 1924)

$$
\text { Let } \Delta \equiv\left|\begin{array}{ll}
\left(A_{m m}\right) & \left(B_{m,}\right) \\
\left(C_{r m}\right) & \left(O_{r r}\right)
\end{array}\right|
$$

where $\left(A_{m m}\right)$ represents a square of $m$ rows and $m$ columns of a's; $\left(B_{m r}\right)$ represents a rectangle of $m$ rows and $r$ columms of $b$ 's; $\left(C_{r, n}\right)$ represents a rectangle of $r$ rows and $m$ columns of $c^{\prime} s$, and $\left(O_{r}\right)$ represents a square of $r$ rows and $r$ columns of zeros.

If now we form a compound determinant $S$ by bordering $\left(A_{m m}\right) \equiv\left|a_{m m}\right| \equiv A$ in all possible ways with $k$ of the remaining rows and columns of $\Delta$ we have by Sylvester's theorem $\dagger$ of 1851

and $\quad S^{\prime}=A^{(r-1)_{k-1}} \quad \Delta^{(r-1)_{k}}$

$$
S=A^{(r-1)_{k}} . \quad \Delta^{(r-1)_{k-1}},\left\{(r-1)_{k}=\frac{(r-1) !}{k !(r-1-k) !}\right\}
$$

where $S^{\prime}$ is the compound determinant formed by bordering $A$ in all possible ways with $(r-k)$ of the remaining rows and columns of $\triangle$; and where the element of $S^{\prime \prime}$ in the position $(i, j)$ is $A$ bordered by the rows and columns not taken for the element in the position $(i, j)$ of $S$.

Let $M_{h}$ represent any minor of $S$ of order $h$ and let $M_{h}^{\prime}$ be the complementary of the corresponding minor in $S^{\prime}$

Then

$$
M_{h}=A^{n-(r-1)_{k-1}} \cdot \Delta^{n-(r-1)_{k}} \cdot M_{h}^{\prime} .
$$

If now $r>m$, then $\Delta=0$, and so $S=S^{\prime \prime}=0$, and $M_{h}=0$, when $h>(r-1)_{k}$.

If $k>m$, then every element of $S$ is zero.

- Crelle's Journal, 72 pp. 152-175.

† Philosophical Magazine (4), i, pp. 295-305. 
If $r-k>m$, then every element of $S^{\prime}$ is zero.

If $k=m$, then every element of $S$ breaks up into two factors, one of which is common to every element in the same row and on removing the common factor for each row the remaining determinant has all its rows identical, from which it follows that every minor of $S$ of order two or greater vanishes.

It is readily seen that when $k=1$ every minor of $S$ ' of order $h, M_{h}$ say, is an example of Sylvester's theorem for a determinant of order $m+h, \Delta^{\prime}$ say, of the same form as $\triangle$, with a square of zeros, $h$ on a side, in the lower right-hand corner.

We have therefore

$$
\mathrm{I}_{h}=\mathbf{A}^{h-1} \cdot \triangle^{\prime}
$$

and when $h>m, \Delta^{\prime}=0$ and therefore $\nu_{h}=0$.

Kronecker's theorem is the case of this when $h=m+1$ and where $\left(B_{m r}\right)$ is $\left(A_{m m}\right)$ with more columns added, and where $\left(C_{r_{m}}\right)$ is $\left(A_{m m}\right)$ with more rows added. 